\title{
A Proof of the Uniform Convergence of the Fourier Series, with Notes on the Differentiation of the Series.
}

\author{
By George A. Gibson, M.A.
}

1. My only justification for presenting this paper to the Society lies in the fact that, so far as I am aware, the uniform convergence of the Fourier Series is nowhere alluded to, and far less discussed, in any English textbook; while the precautions that are necessary in differentiating the series are hardly ever mentioned even in treatises which give a very thorough treatment of its convergence. I have confined myself almost exclusively to what may be called ordinary functions, as a complete discussion of what has been done in recent years for functions that lie outside the category of "ordinary" would make the paper much too long. For information as to the original authorities, I would refer to the paper which I communicated to the Society last session On the History of the Fourier. Series. It is sufficient to say here that the proof $I$ now give is simply an adaptation of that of Heine (Kugelfunctionen, Bd. I. $57-64$, Bd. II. 346-353) and of that of Neumann (Über die nach Kreis . . Functionen fortsch. Entwickelungen, 26-52).

2. The chief instrument employed in the investigation is The Second Theorem of Mean Value. A sketch of a proof of this theorem will be found in a paper by me in Vol. VI., pp. 40-42 of the Society's Proceedings; but for a satisfactory treatment of this and other theorems of the Integral Calculus I would refer to Cathcart's translation of Harnack's Introduction to the Calculus. The theorem, so far as we are now concerned with it, may be stated thus:-If, for all values of $x$ between $a$ and $b, f(x)$ and $\phi(x)$ are finite integrable functions of $x$ and if $f(x)$ is either not increasing or not decreasing (i.e., has no turning. points) in the interval $(a, b)$, then

$$
\left.\begin{array}{c}
\int_{a}^{b} f(x) \phi(x) d x=f(a) \int_{a}^{\xi} \phi(x) d x+f(b) \int_{\xi}^{b} \phi(x) d x \quad a \leqq \xi \leqq b \\
=f(a) \int_{a}^{b} \phi(x) d x+\{f(b)-f(a)\} \int_{\xi}^{b} \phi(x) d x .
\end{array}\right\}
$$


It is to be understood thiat $f(a)=f(a+0)=\mathrm{L}_{\epsilon=0} f(a+\epsilon)$ and $f(b)=f(b-0)=\operatorname{L}_{\epsilon=0} f(b-\epsilon)$ where $a<b$ and $\epsilon$ is positive.

3. When a single-valued function $f(x)$ is said to be given arbitrarily in the interval $(a, b)$, then to each value of $x$ corresponds one value of $f(x)$ in such a manner that the value of the function for one value of the argument in no way conditions its value for another value of the argument. An arbitrary function of this kind is, however, too general for representation by a Fourier (or any other) series, and for the present paper we subject the function to the following restrictions, though some of them may be removed and a Fourier representation of the function still exist. The argument $x$ is, merely for convenience, restricted to the interval $(-\pi, \pi)$.

(i.) $f(x)$ is to be finite, having an upper limit G to its numerical values; (ii.) it is to be continuous except for a finite number of values of $x$ for which it may be discontinuous, but only so that $f(x+0)$ and $f(x-0)$ are each definite, though unequal; (iii.) it is to have a finite number of maxima and minima-i.e., a finite number of turning points. Such a function is necessarily integrable.

$$
\text { 4. Let } \quad \mathrm{S}_{2 n+1}=\frac{1}{2} \mathrm{~A}_{0}+\sum_{r=1}^{n}\left(\mathrm{~A}_{r} \cos r x+\mathrm{B}_{,} \sin r x\right)
$$

where $\mathrm{A}_{r}=\frac{1}{\pi} \int_{-\pi}^{\pi} f(\alpha) \cos r a d a, \quad \mathrm{~B}_{r}=\frac{1}{\pi} \int_{-\pi}^{\pi} f(\alpha) \sin r a d a$.

Hence $\mathbf{S}_{: n+1}=\frac{1}{\pi} \int_{-\pi}^{\pi} f(\alpha)\left\{\frac{1}{2}+\sum_{r=1}^{n} \cos r(\alpha-x)\right\} d \alpha$

$$
\left.\begin{array}{l}
=\frac{1}{\pi} \int_{-\pi}^{\pi} f(a) \frac{\sin (2 n+1) \frac{a-x}{2}}{2 \sin \frac{\alpha-x}{2}} d a \\
=\frac{1}{\pi} \int_{0}^{\frac{\pi-x}{2}} f(x+2 \alpha) \frac{\sin (2 n+1) \alpha}{\sin \alpha} d a \\
+\frac{1}{\pi} \int_{0}^{\frac{\pi+x}{2}} f(x-2 \alpha) \frac{\sin (2 n+1) a}{\sin \alpha} d a
\end{array}\right\}
$$


It has to be shown that, excluding values of $x$ in the neighbonerhood* of the points of discontinuity, though not these points themselves, a finite value of $n$ can be found, which is the same for every $x$, such that the difference between $S_{2 n+1}$ and $1_{2}\{f(x+0)+f(x-0)\}$ shall be as small as we please, except that when $x= \pm \pi$ the value $\frac{1}{2}\{f(-\pi+0)+f(\pi-0)\}$ is to be taken instead of $\frac{1}{2}\{f(x+0)$ $+f(x-0)\}$. If $f(\pi-0) \neq f(-\pi+0)$, the points $x= \pm \pi$ are to be reckoned points of discontinuity. In other words, the convergence of the series has to be shown to be in general uniform.

The demonstration is divided into two parts, in the first of which it is shown, inter alia, that the coefficients $\mathbf{A}_{w}, \mathbf{B}_{n}$ have zero for their limit.

5. Consider the integral $\int_{a}^{b} \phi(a) \sin m a d a$ where $m$ is any positive number, integral or fractional, and $a, b$ and $\phi(a)$ may contain a parameter $x$ (e.g., $\phi(\alpha)$ might be $\phi(x+2 \alpha)$ ) and $\phi(\alpha)$ is subject to the same restrictions as $f(x)$ in $\$ 3$.

Let $a_{1}, a_{2}, \ldots a_{p}$ be the turning points or points of discontinuity of $\phi(\alpha), p$ in number and let $G$ be an upper linit to the numerical values of $\phi(a)$. Divide the interval $(a, b)$ into the $p+1$ partial intervals $\left(a, a_{1}\right) \ldots\left(a_{r}, a_{r+1}\right) \ldots\left(a_{p}, b\right)$ and take the integral for the interval $\left(a_{r}, a_{r+1}\right)$ to which theoren (1) is applicable.

$$
\begin{aligned}
\int_{a_{r}}^{a_{r+1}} \phi(a) \sin m a d a & =\phi\left(a_{r}\right) \int_{a_{r}}^{\xi} \sin m a d a+\phi\left(a_{r+1}\right) \int_{\xi}^{a_{r+1}} \sin m \alpha d a ; a_{r} \approx \xi<a_{r+1} \\
& =\phi\left(a_{r}\right) \frac{\cos m a_{r}-\cos m \xi}{m}+\phi\left(a_{r+1}\right) \frac{\cos m \xi-\cos m a_{r+1}}{m} \\
& <\frac{2}{m}\left\{\phi\left(a_{r}\right)+\phi\left(a_{r+1}\right)\right\}<\frac{4 G}{m} .
\end{aligned}
$$

$\therefore \int_{a}^{b} \phi(\alpha) \sin m a r a<\frac{4(p+1) G}{m}$ in absolute value.

Now $m$ can be chosen so large that $4(p+1) \mathrm{G} / m$ shall be as small as we please, and this independently of the parameter $x$; in other words, the integral converges uniformly to zero with $1 / m$.

" See 86 for a definition of "neighbourhood of a point." 
In the same way it may be shown that $\int_{a}^{b} \phi(\alpha) \cos m a d a$ converges uniformly to zero with $1 / \mathrm{m}$.

These results show that the coefficients of the series, given by (2), are ultimately zero. It should be observed, however, that $A_{n}, B_{n}$ are in general only of the order $1 / n$; it is only in exceptional cases, as will be seen later, that they are of the order $1 / n^{2}$. Hence the series is usually semi-convergent, while the series obtained by differentiating a Fourier series term by term will usually not be convergent.

The presence of the factor $p+1$ in (4) should be noted as an indication of the necessity for the restrictions (ii.) and (iii.) of $\$ 3$.

We may also deduce another result that will be useful immediately. If we suppose $\mathbf{Q}>\mathbf{P}>0$, then

$$
\int_{0}^{\mathrm{Q}} \frac{\sin \alpha}{\alpha} d \alpha-\int_{0}^{\mathrm{P}} \frac{\sin \alpha}{\alpha} d \alpha=\int_{\mathrm{P}}^{\mathrm{Q}} \frac{\sin \alpha}{\alpha} d \alpha<\frac{2}{\mathrm{P}}+\frac{2}{\mathrm{Q}} \text { numerically. }
$$

But we know that the value of the first integral for $Q=\infty$ is $\pi / 2$. Hence if $\mathbf{P}>0$

$$
\int_{0}^{1} \frac{\sin \alpha}{\alpha} d a<\frac{\pi}{2}+\frac{2}{\mathrm{P}} \text { in absolute value. }
$$

6. By means of equations (4) and (5) we can tind limits for each of the integrals in (3).

Let an arbitrarily small but finite distance $\delta$ be measured off on each side of the points for which the function is discontinuous, thus forming what we may call the neighbourhood of the points; the points $\pm \pi$ are also to be dealt with in the same way. A point is thus in the neighbourhood of $c$ if its abscissa lies between $c-\delta$ and $c+\delta$. Then suppose in the first place, $-\pi+\delta=x<\pi-\delta$ and let $x$ have any value in this range except those in the neighbourhood of the points of discontinuity, though it may have exactly a value for which the function is discontinuous.

We shall now show that, given an arbitrarily small quantity $2 \epsilon$, we can choose $n$ so large, but finite, that $S_{2 n+1}$ shall differ from $\frac{1}{2}\{f(x+0)+f(x-0)\}$ by a quantity that is less than $2 \epsilon / \pi$ and that such $n$ is the same for every $x$. 
Consider $\quad \int_{0}^{\frac{\pi-x}{2}} f(x+2 \alpha) \frac{\sin m a}{\sin \alpha} d a \quad$ where $\quad m=2 n+1$,

although the particular form of $m$ is of no moment; it may be either integral or fractional. Put $\psi(x, \alpha)=(\alpha / \sin a) f(x+2 \alpha)$, so that $\psi(x, 0)$ is equal to $f(x+0)$. Take $\eta$ an arbitrarily small but finite quantity, such that $0<\eta \leqq(\pi-x) / 2$. Then

$$
\begin{gathered}
\int_{0}^{\frac{\pi-x}{2}} f(x+2 \alpha) \frac{\sin (2 n+1) \alpha}{\sin \alpha} d \alpha=\int_{0}^{\frac{\pi-x}{2}} \psi(x, a) \frac{\sin m \alpha}{a} d \alpha \\
=\int_{0}^{\eta} \psi(x, \alpha) \frac{\sin m a}{\alpha} d \alpha+\int_{\eta}^{\frac{\pi-x}{2}} \psi(x, \alpha) \frac{\sin m \alpha}{\alpha} d \alpha
\end{gathered}
$$

To the last of these integrals equation (4) is applicable if we denote by $\mathrm{G}$ the greatest value of $\psi(x, a)$ and then write $\mathrm{G} / \eta$ in place of $\mathrm{G}$ of $(4)$, since $\mathrm{G} / \eta$ will be the greatest value of $\psi(x, a) / \alpha$. Hence

$$
\int_{\eta}^{\frac{\pi-2}{2}} \psi(x, a) \frac{\sin m a}{u} d a<\frac{\left(x^{\prime}\right.}{m \eta} \quad \text { numerically }
$$

where $\mathrm{G}^{\prime}$ is a finite constant independent of $x$, though depending on $p$.

Again, no matter what the value of $x$, we can choose $\eta$ so small but finite that $\psi(x, a)$ shall have no turning point for any value of $a$ between 0 and $\eta$; hence we can apply equation (1) to the first integral on the right of $(6)$. Thus

$$
\begin{gathered}
\int_{0}^{\eta} \psi(x, \alpha) \frac{\sin m \varepsilon \alpha}{\alpha} d \alpha=\psi(x, 0) \int_{0}^{\eta} \frac{\sin m a}{\alpha} d a \\
+\{\psi(x, \eta)-\psi(x, 0)\} \int_{\xi}^{\eta} \frac{\sin m a}{\alpha} d a
\end{gathered}
$$

and therefore

$$
\int_{0}^{\eta} \psi(x, a) \frac{\sin m a}{a} d \alpha=f(x+0) \int_{0}^{m \eta} \frac{\sin \beta}{\beta} d \beta+\{\psi(x, \eta)-\psi(x, 0)\} \int_{m \xi}^{m \eta} \frac{\sin \beta}{\beta} d \beta
$$

where $0 \equiv \xi \equiv \eta$. 
Further, we can choose $\eta$ so small, but finite, that $\psi(x, \eta)-\psi(x, 0)$ shall for every $x$ be less than any arbitrarily small quantity $\epsilon^{\prime}$, while $\int_{m \xi}^{m \eta} \frac{\sin \beta}{\beta} d \beta \ngtr \int_{0}^{\infty} \frac{\sin \beta}{\beta} d \beta \ngtr \frac{\pi}{2}$. Hence by (5) we may now write (8)

$$
\begin{aligned}
& \int_{0}^{\eta} \psi(x, a) \frac{\sin m a}{\alpha} d a<f(x+0)\left(\frac{\pi}{2}+\frac{2}{m \eta}\right)+\frac{\pi}{2} \epsilon^{\prime} \\
&<\frac{\pi}{2} f(x+0)+\frac{2 \mathrm{G}}{m \eta}+\frac{\pi}{2} \epsilon^{\prime} \\
& f(x+0)<\mathrm{G} .
\end{aligned}
$$

since

By means of (7) and (9) we can now write (6) thus

$$
\begin{aligned}
& \int_{0}^{\frac{\pi-x}{2}} f(x+2 \alpha) \frac{\sin (2 n+1) a}{\sin \alpha} d \alpha<\frac{\pi}{2} f(x+0)+\frac{\mathrm{G}^{\prime}}{m \eta}+\frac{2 \mathrm{G}}{m \eta}+\frac{\pi}{2} \epsilon^{\prime} \\
& <\frac{\pi}{2} f(x+0)+\frac{G^{\prime \prime}}{(2 n+1) \eta}+\frac{\pi}{2} \epsilon^{\prime}
\end{aligned}
$$

where $G^{\prime \prime}$ is a finite constant independent of $x$.

The theorem to be proved is now obvious. Suppose $\epsilon$ given, we first choose $\epsilon^{\prime}$ so that $\pi \epsilon^{\prime} / 2$ is less than $\epsilon / 2$; we then choose $\eta$ to satisfy this value of $\epsilon^{\prime} ; n$ is then chosen so large that $G^{\prime \prime} /(2 n+1) \eta$ shall also be less than $\epsilon / 2$. The right-hand side of $(10)$ is then less than $\frac{\pi}{2} f(x+0)+\epsilon$.

The same considerations apply to the second integral in (3) so that we have $\mathrm{S}_{2 n+1}<\frac{1}{2}\{f(x+0)+f(x-0)\}+\frac{2 \epsilon}{\pi}$ and the larger of the two values of $n$ thus determined is the one required. In other words, $\underset{n=\infty}{\mathbf{L}} \mathbf{S}_{2 n+1}=\frac{1}{2}\{f(x+0)+f(x-0)\}$ and the convergence is uniform.

7. Suppose now, in the second place, that $x$ is in the neighbourhood of a point of discontinuity. From equations (6) and (7) it is evident that the integrals whose limits are $0,(\pi-x) / 2$ and $0, \eta$ 
respectively converge to the same value, as $m$ becomes infinite, even if $\eta$ at the same time converge to zero, provided only that $m \eta$ at the same time becomes infinite ; this would be the case if, e.g., $\eta=1 / \sqrt{ } m$. This result is at first sight a little startling, though it is by no means without parallel in the theory of limiting values. In the case now under consideration it will contribute greatly to clearness to draw the curve $y=f(x+2 a)$ with $a$ for abscissa, giving to $x$ values that lie within the neighbourhood of a point of discontinuity. Let $c$ be a value for which $f(x)$ is discontinuous, and consider values of $x$ lying between $c-\delta$ and $c-0$. Suppose $x=c-\delta$; then when $a=0, y=f(c-\delta)$, As $a$ increases from 0 to $\delta / 2, y$ changes from $f(c-\delta)$ to $f(c-0)$. In forming the integrals $(6)$ for the values $c-\delta$ of $x$, the greatest value one would give to $\eta$ would be $\delta / 2$, in order that the interval $(0, \eta)$ should not contain the point of discontinuity, though of course $\eta$ might require to be smaller in order to satisfy the conditions respecting $\epsilon^{\prime}$ and $\epsilon$, in the equations corresponding to (8), (9), (10). Again, if $c-\delta<x<c$, then $\eta$ would not be so large as $\delta / 2$, and as $x$ approaches indefinitely near to $c, \eta$ becomes indefinitely small, and therefore $m$ must be taken indefinitely great in order that $m \eta$ may also be indefinitely great. The convergence thus becomes infinitely slow for the part of the series depending on $f(x+2 a)$ and we can assign no finite value for $m$ such that for every $x$ in the neighbourhood of $c$ the value of $1 / m \eta$ shall become arbitrarily small. In other words, while by equations (6) and (7) in virtue of the observation made at the beginning of this paragraph we can for any given $x$ find a value of $m$ which will make the series converge, we must when we take a new value of $x$ usually take a new value of $m$, and $m$ becomes ultimately indefinitely great; that is, the series does not converge uniformly in the neighbourbood of the point of discontinuity. These considerations do not, however, apply if $x=c$; the convergence of each of the integrals is sinply of the nature of the cases of $\$ 6$. Even in the case of non-uniform convergence, it is only one of the integrals in (3) that falls under the class considered in this paragraph. It is of course known from general principles that when a series represents a discontinuous function the convergence is non-uniform in the neighbourhood of the points of discontinuity ; but I have thought it better to deduce the result in this case from the analysis used to establish the general theorem.

4 Vol. 12 
8. The values $\pm \pi$ have still to be considered. For these the reasoning may be gone over afresh; but it is simpler to suppose $f(x)$ continued for values of $x$ greater than $\pi$ and less than $-\pi$, so that $f(x+2 \pi)=f(x)$ and therefore $f^{\prime \prime}(\pi+0)=f(-\pi+0)$ and $f(-\pi-0)=f(\pi-0)$. If the origin from which $x$ is measured be then shifted, these points become ordinary points or points of discontinuity like those previously discussed; hence in the neighbourhood of $\pm \pi$, if $f(\pi) \neq f(-\pi)$ the convergence is not uniform, and if $x= \pm \pi$ the value to which $S_{3 n+1}$ converges is $\frac{1}{2}\{f(-\pi+0)+f(\pi-0)\}$ which of course reduces to $f(\pi)$ if $f(\pi)=f(-\pi)$.

9. There is one point in the proof just given that perhaps deserves notice. Is it quite clear that the neighbourhood of points at which the function is a maximum or a minimum is not of the same character as that of a point of discontinuity? Must not $\eta$ for such points diminish and $m$ increase beyond all limit as $x$ approaches the value for which the function has a turning point? Whatever difficulty may seem to exist is at once removed by the observation of Heine that if $f^{\prime \prime}(c)$ be a maximum value, then, between the two adjacent minima that include $f^{\prime \prime}(c), f^{\prime \prime}(x)$ can be put in the form $\phi(x)+\psi(x)$

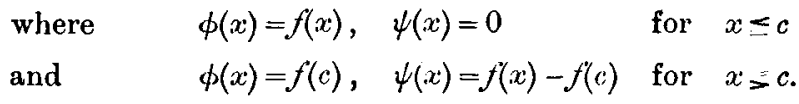

Evidently $\phi /(x)$ is not decreasing and $\psi(x)$ not increasing, so that to each the Mean Value Theorem is applicable. Turning points are thus of the same nature as ordinary points. Heine's treatment of the Fourier series based on the representation of a function as the sum of functions which are either not increasing or not decreasing is very instructive.

10. Equations (6) and (7) establish Riemann's Theorem for functions of the kind we are considering, namely, that the value of the series at any point depends only on the behaviour of the function in the neighbourhood of the point. In establishing this result and the convergence generally, the integral $\int_{0}^{h} \phi(\alpha) \cdot \sin m \alpha \cdot d \alpha / \mu$, first thoroughly discussed by Dirichlet, plays a leading part and has been appropriately called Dirichlet's Integral by Kronecker. 
11. The restrictions imposed on $f(x)$, generally called Dirichlet's conditions, ${ }^{*}$ still leave a great amount of arbitrariness to it; when we seek to remove restrictions the investigation begins to get rather complicated. Some of them however can be removed, in part at least, by considerations founded simply on the nature of an integral. Suppose, for instance, that for $x=c, f(x)$ is infinite but $(x-c)^{r} f(x)$ finite and definite when $x=c, r$ being positive and less than unity. $f(x)$ would still be integrable over the whole range, and excluding the neighbourhood of $c$ the series would still converge for all other values as before. Functions which oscillate indefinitely often in the neighbourhood of a finite number of points can be dealt with in the same way. But cases like these are of comparatively little practical importance. Instances of trigonometric series in which the function is intinite for particular values of the argument are conımon enough in analysis. (See, e.g., Chrystal's Algebra II., p. 310, ex. 13, 14.)

12. It is now obvious that the Fourier series can be integrated and therefore that the expansion is unique, when the function is supposed to possess the properties laid down in $\$ 3$ for the whole interval. The resulting series will not however, in general, be a Fourier series since the term $\frac{1}{2} A_{0}$ will introduce the term $\frac{1}{2} A_{0} x$. The case is different when we consider the differentiation of the series. We suppose the differential coefficient $f^{\prime}(x)$ to have the properties required by $\S 3$.

The first theorem to be given is this: $-f^{\prime}(x)$ will only be obtained by differentiating the series for $f(x)$ term by term if $f(x)$ be throughout continuous and besides $f(\pi)=f(-\pi)$.

Suppose

$$
\begin{aligned}
& f(x)=\frac{1}{2} \mathrm{~A}_{0}+\Sigma \mathrm{A}_{n} \cos n x+\Sigma \mathrm{B}_{n} \sin n x \\
& f^{\prime}(x)=\frac{1}{2} \mathrm{C}_{v}+\Sigma \mathrm{C}_{n} \cos n x+\Sigma \mathrm{D}_{n} \sin n x .
\end{aligned}
$$

If we calculate the values of $\mathrm{C}_{n}, \mathrm{D}_{n}$ we find

$$
\mathrm{C}_{0}=0 \quad \mathrm{C}_{n}=\imath \mathrm{B}_{v}, \quad \mathrm{D}_{n}=-n \mathrm{~A}_{n}
$$

so that $f^{\prime}(x)$ is got by differentiating the series (11).

"It is to be understond that the addition "that $f(x)$ may become infinite" is not included, so that these are Dirichlet's conditions in the narrower acceptation of the phrase. 
Suppose however that $f(x)$ is continuous but $f(\pi) \neq f(-\pi)$; we then get

$$
\mathrm{C}_{0}=\frac{f(\pi)-f(-\pi)}{\pi}, \quad \mathrm{C}_{n}=\frac{f(\pi)-f(-\pi)}{\pi} \cos n \pi+n \mathrm{~B}_{n}, \quad \mathrm{D}_{n}=-n \mathrm{~A}_{n}
$$

so that $f^{\prime}(x)$ even in this simple case is not got by differentiating (11) as it stands.

Denoting $\{f(\pi)-f(-\pi)\}$ by $\mathrm{C}$ and expanding $\frac{1}{2} \mathrm{C} x$ into the Fourier series $-C \Sigma \frac{\cos n \pi}{n} \sin n x$ we can write (11) in the form

$$
f(x)=\frac{1}{2} \mathrm{~A}_{0}+\frac{1}{2 \pi} \mathrm{C} x+\Sigma \mathrm{A}_{n} \cos n x+\mathcal{(}\left(\mathrm{B}_{n}+\frac{\mathrm{C} \cos n \pi}{n \pi}\right) \sin n x .
$$

The differentiation of this series gives the proper value for $f^{\prime}(x)$. In (11) the value of $\mathrm{B}_{n}$ is $-\mathrm{C} \cos n \pi / n \pi+\mathrm{C}_{n} / n$, so that the series obtained by differentiating (11) will usually be oscillating, or divergent. The procedure adopted in using the form (13) may be compared with that of representing the product series for $\sin x$ in the form

$$
\sin \pi x=\pi x \Pi\left(1+\frac{x}{n}\right) e^{-\frac{x}{4}}
$$

13. Suppose now that $f(x)$ is discontinuous for $x=c$ and put $f(c+0)-f(c-0)=\mathrm{D}$. Take a function $\phi(x)=f(x)+\mathrm{D} \theta(x)$ where

and

$$
\theta(x)=1 \text { from } x=-\pi \text { to } x=c-0
$$

$$
\theta(x)=0 \text { from } x=c+0 \text { to } x=\pi \text {. }
$$

The function $\phi(x)$ is continuous in the neighbourhood of $c$, since $\phi(c-0)=f(c+0)=\phi(c+0)$. Moreover $\phi(x)$ has the same differential coefficient as $f^{\prime}(x)$. The Fourier expansion for $\phi(x)$ is, using $A_{n}, B_{n}$ to denote the cofficients in (11),

$$
\begin{aligned}
& \phi(x)=\frac{1}{2}\left\{\mathrm{~A}_{0}+\frac{\pi+c}{\pi} \mathrm{D}\right\}+\mathcal{L}\left\{\mathrm{A}_{u}+\frac{\mathrm{D} \sin n c}{n \pi}\right\} \cos \iota x \\
& +\mathcal{Z}\left\{\mathrm{B}_{n}+\frac{\mathrm{D}(\cos n \pi-\cos n c)}{n \pi}\right\} \sin n x \text {. }
\end{aligned}
$$

Before we can differentiate we must take account of the values for $\pm \pi$. Denoting $f(\pi)-f(-\pi)$ by $\mathrm{D}^{\prime}$ we have

$$
\phi(\pi)-\phi(-\pi)=\mathrm{D}^{\prime}-\mathrm{D}
$$


and the corresponding equation to (11) will be

$$
\begin{aligned}
& \phi(x)=\frac{1}{2}\left\{\mathrm{~A}_{0}+\frac{\pi+c}{\pi} \mathrm{D}\right\}+\frac{1}{2 \pi}(\mathrm{D}-\mathrm{D}) x+\Sigma\left\{\mathrm{A}_{n}+\frac{\mathrm{D} \sin n c}{n \pi}\right\} \cos n x \\
& +\Sigma\left\{B_{n}-\frac{D \cos n c}{n \pi}+\frac{D^{\prime} \cos n \pi}{n \pi}\right\} \sin n x
\end{aligned}
$$

If we differentiate (15) term by term we get the value of the series (12), as may be readily proved by going through the necessary differentiations and integrations.

In the same way if $f(x)$ were also discontinuous for $x=c_{1}$, we should put $\phi(x)=f(x)+\mathrm{D} \theta(x)+\mathrm{D}_{1} \theta_{1}(x)$ where $\mathrm{D}$ and $\theta(x)$ are as before, $D_{1}=f\left(c_{1}+0\right)-f\left(c_{1}-0\right)$ and

$$
\begin{array}{lll}
\theta_{1}(x)=1 & \text { from } x=-\pi & \text { to } x=c_{1}-0 \\
\theta_{1}(x)=0 & \text { from } x=c_{1}+0 & \text { to } x=\pi .
\end{array}
$$

The equation corresponding to (15) would then be formed and so on for any number of points of discontinuity.

The procedure of this paragraph was suggested by the method of Heine referred to in $\$ 9$, although Heine does not discuss the differentiation of the series except in regard to the cases mentioned in $\$ 12$.

14. It may perhaps be of interest to give some examples. In dealing with series which represent a function that is given only for a portion of the interval $(-\pi, \pi)$, it is important to bear in mind that we may have an infinite number of series which for that portion coincide with the function, each series being determined by the way in which we suppose the function continued beyond the given portion so as to be detined for the complete interval $(-\pi, \pi)$. The most frequently occurring cases are those for which (i.) $f(-x)=f(x)$ and (ii.) $f(-x)=-f(x)$, giving rise to cosine and to sine series respectively.

Take the two series

$$
\begin{aligned}
& e^{a x}=\frac{2}{\pi} \sum_{l}^{\infty} \frac{n}{a^{2}+n^{2}}\left(1-e^{a \pi} \cos n \pi\right) \sin n x \\
& e^{a}=\frac{e^{a \pi}-1}{a \pi}+\frac{2 a}{\pi} \sum_{1}^{\infty} \frac{e^{a \pi} \cos n \pi-1}{a^{2}+n^{2}} \cos n x .
\end{aligned}
$$

If we differentiate (17) term by term we get (16), but we do not get (17) by differentiating (16); on the other hand, we should get (17) 
by integrating (16) term by term, but we should not, immediately at least, get (16) by integrating (17) term by term. The reason for the difference in behaviour is obvious after what has been said. Equation (16) is deduced on the understanding that in the interval $(0, \pi) f(x)=e^{a x}$ and in the interval $(-\pi, 0) f(x)=-e^{-a x}$; we have therefore $f(\pi)-f(-\pi)$ equal to $2 e^{a \pi}=\mathrm{D}^{\prime}$ while $f(+0)=1$ $f(-0)=-1$ and $\therefore f(+0)-f(-0)=2=\mathrm{D}$. Equation (16) must therefore be put in the form (15), namely

$$
\phi(x)=1+\frac{e^{a \pi}-1}{\pi} x+\frac{2}{\pi} \geq \frac{e^{a \pi} \cos n \pi-1}{n} \cdot \frac{a^{2}}{a^{2}+n^{2}} \sin n x
$$

and this equation when differentiated yields (17).

Equation (17), on the other hand, is obtained on the understanding that in the interval $(0, \pi) f(x)=e^{a x}$ and in the interval $(-\pi, 0) f(x)=e^{-a x}$, so that $f(\pi)=f(-\pi)$ and $f(+0)=f(-0)$. By $\S 12$ it follows that $f^{\prime}(x)$ is obtained by differentiating (17) as it stands.

Suppose again we have

$$
\cos x=\frac{2}{\pi} \sum_{1}^{\infty} \frac{1+(-1)^{n}}{n^{2}-1} n \sin n x
$$

In this case $f(x)=\cos x$ in the interval $(0, \pi)$ but $f(x)=-\cos x$ in interval $(-\pi, 0)$. Hence

$$
f(\pi)-f(-\pi)=-2=\mathrm{D}^{\prime}, f(+0)-f(-0)=\hat{z}=\mathrm{D} .
$$

Hence the form corresponding to $(15)$ is

$$
\phi(x)=1-\frac{2 x}{\pi}+\frac{4}{\pi} \underset{1}{\stackrel{\infty}{\infty}} \frac{\sin 2 n x}{2 n\left(4 n^{2}-1\right)}
$$

from which we obtain

$$
\sin x=\frac{2}{\pi}-\frac{4}{\pi} \sum_{1}^{\infty} \frac{\cos 2 n x}{4 n^{2}-1}
$$

These examples are sufticient to show the care necessary in differentiating infinite series.

It is perhaps not out of place to add that the most natural method of passing to Fourier's Integral Formulae is to start from the equations of $\$ \$ 5$ and 6 , as is done by Neumann. 